\section{Rac-king up new roles in haematopoiesis}

Rac1 and Rac2 guanosine triphosphatases (GTPases) are crucial signalling regulators in eukaryotic cells, acting downstream of various cellular receptors. Two studies published in Science have used conditional gene targeting to clarify the roles of Rac1 and Rac2 in particular aspects of haematopoietic-cell differentiation.

Gu et al. looked at the differential activity of Rac1 and Rac2 in haematopoietic stem/progenitor cells (HSC/Ps). As Racl deficiency is embryonic lethal, they generated conditional Rac1-knockout mice. Deficiency of Racl inhibited the ability of HSC/Ps to reconstitute haematopoiesis in an engraftment model (which requires adhesion and proliferation in the bone marrow), and the absence of both Rac1 and Rac2 led to the movement of progenitor cells out of the bone marrow and into the peripheral circulation, as a result of decreased adhesion to fibronectin. Rac1 and Rac2 were also shown to have roles in regulating the cytoskeletal changes that are required for engraftment and mobilization.

$\mathrm{RaCl}^{-1-}$, and to a greater extent $\mathrm{Rac1}^{-/} \mathrm{Rac2}^{--}$, HSC/Ps had reduced proliferation in response to growth factors in vitro, correlating with decreased levels of cyclin D1 and phosphorylation of extracellular signal-regulated kinase (ERK), and increased levels of the cyclindependent kinase inhibitor KIP1. By contrast, Rac2 $^{-/-}$cells were more susceptible to apoptosis after growthfactor stimulation, associated with reduced Akt activation. Therefore, it seems that Rac1 regulates entry into the cell cycle, whereas Rac2 mainly regulates cell survival.

Walmsley et al. looked further downstream at B-cell development by generating, for the first time, mice with a conditional deletion of Rac1 in the B-cell lineage ( $R a c 1^{\mathrm{B}}$ mice). $\mathrm{Racl}^{\mathrm{B}} \mathrm{Rac2}^{-/-}$mice had normal numbers of pro-B, pre-B and immature $\mathrm{B}$ cells in the bone marrow, but reduced numbers of $B$ cells at later developmental stages in the spleen and lymph nodes. In the absence of both Rac1 and Rac2, B-cell development seems to be blocked at the transitional type $1 \mathrm{~B}$-cell stage in the spleen.

Recent studies have shown activation of Racl after B-cell receptor (BCR) stimulation, and signals from the BCR are known to be crucial for B-cell development, so are BCR signalling defects responsible for the observed phenotype of $\mathrm{Racl}^{\mathrm{B}} \mathrm{Rac2^{-/- }}$ mice? Rac2 ${ }^{-/-}$and $\mathrm{Racl}^{-/+} \mathrm{Rac2}^{-/-}$ mature B cells stimulated with an IgM-specific antibody had decreased survival compared with wild-type B cells, which correlated with decreased induction of the antiapoptotic protein $\mathrm{Bcl}-\mathrm{X}_{\mathrm{L}}$. These $B$ cells also proliferated less than wild-type cells. This was shown to be due to decreased induction of the cell-cycle regulator cyclin D2 after stimulation. So, Rac1 and Rac2 transduce BCR signals for B-cell survival and entry into the cell cycle. Finally, in contrast to wild-type cells, BAFF could not increase the survival of $\mathrm{Racl}^{\mathrm{B}} \mathrm{Rac2}^{-/-}$immature B cells, and this was probably the result of decreased expression of BAFF receptor (BAFFR). Rac1 and Rac2 were shown to transduce BCR signals leading to the upregulation of expression of BAFFR messenger RNA.

These two studies show that in both B cells and HSC/Ps, Racl and Rac2 have essential roles in controlling proliferation and survival. Furthermore, despite their sequence similarity, the two GTPases seem to have partially non-redundant physiological functions. Gu et al. confirmed this functional distinction in neutrophils, in which Rac2 seems to be the main GTPase regulating directed migration and superoxide generation, whereas Rac1 regulates neutrophil shape.

Kirsty Minton

(2) References and links ORIGINAL RESEARCH PAPERS Gu, Y. et al. Hematopoietic cell regulation by Rac1 and Rac2 guanosine triphosphatases. Science $\mathbf{3 0 2}$, 445-449 (2003) | Walmsley, M. J. et al. Critical roles for Rac1 and Rac2 GTPases in B cell development and signaling. Science $\mathbf{3 0 2}$ 459-462 (2003)

\section{IN BRIEF}

\section{NATURAL KILLER CELLS}

The mature activating natural killer cell immunologic synapse is formed in distinct stages.

Orange, J. S. et al. Proc. Natl Acad. Sci. USA 100, 14151-14156 (2003)

The interface between a natural killer (NK) cell and its target cell — the activating NK-cell immunological synapse — is highly organized. In this study, Orange et al. show that CD2 and CD11b, similar to CD11a, co-localize with filamentous actin in the peripheral supramolecular activation cluster (pSMAC), whereas perforin accumulates in the central SMAC (cSMAC). Polarization of CD2, CD11a and CD11b to the pSMAC was dependent on actin polymerization and Wiskott-Aldrich syndrome protein (WASP), but independent of microtubule function. By contrast, perforin accumulation in the cSMAC was dependent on actin polymerization, WASP and microtubule function. In addition, the rate of $\mathrm{CSMAC}$ perforin accumulation was slower than the recruitment of receptors to the pSMAC, indicating that formation of the NK-cell immunological synapse is a highly regulated, sequential process.

\section{MUCOSAL IMMUNOLOGY}

Spheniscins: avian $\beta$-defensins in preserved stomach contents of the king penguin, Aptenodytes patagonicus.

Thouzeau, C. et al. J. Biol. Chem. 2 October 2003 (doi:10.1074/jbc.M306839200)

In this paper, Thouzeau and colleagues have identified two new avian antimicrobial peptides in the stomach of the male king penguin. These peptides, known as spheniscin 1 and 2, belong to the $\beta$-defensin family and have broad antimicrobial activity against pathogenic bacteria and fungi. The levels of spheniscin 1 and 2 are higher during periods of food storage than when the birds are digesting, and interestingly, a drop in spheniscin levels correlated with a change from food storage to digestion. These antimicrobial peptides act to protect the surface of the bird's gastrointestinal tract from damage or invasion, and might be important for the long-term preservation of stored food, which can be important for chick survival.

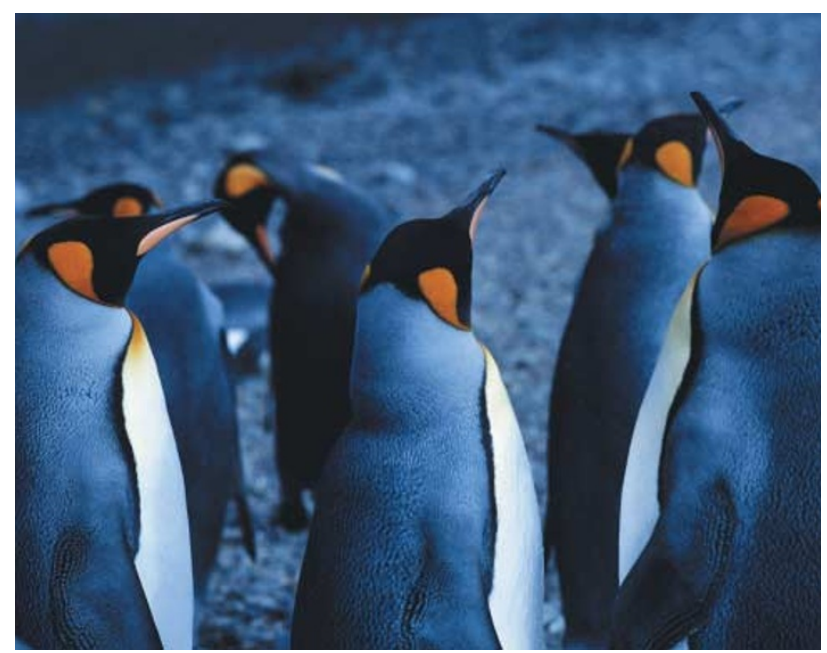

